Canadian

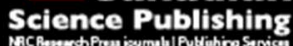

Applied Physiology, Nutrition, and Metabolism Physiologie appliquée, nutrition et métabolisme

\title{
The Modification of the Ketogenic Diet Mitigates Its Stunting Effects in Rodents
}

\begin{tabular}{|c|c|}
\hline Journal: & Applied Physiology, Nutrition, and Metabolism \\
\hline Manuscript ID & apnm-2017-0374.R1 \\
\hline Manuscript Type: & Article \\
\hline Date Submitted by the Author: & 16-Aug-2017 \\
\hline Complete List of Authors: & $\begin{array}{l}\text { Liśkiewicz, Arkadiusz; Medical University of Silesia School of Medicine in } \\
\text { Katowice, Department of Physiology; The Jerzy Kukuczka Academy of } \\
\text { Physical Education, Faculty of Physiotherapy, Laboratory of Molecular } \\
\text { Biology } \\
\text { Kasprowska-Liśkiewicz, Daniela; The Jerzy Kukuczka Academy of Physical } \\
\text { Education, Faculty of Physiotherapy, Laboratory of Molecular Biology; } \\
\text { Medical University of Silesia, School of Medicine in Katowice, Department } \\
\text { for Experimental Medicine } \\
\text { Sługocka, Anna; Medical University of Silesia, School of Medicine in } \\
\text { Katowice, Department for Experimental Medicine } \\
\text { Nowacka-Chmielewska, Marta; The Jerzy Kukuczka Academy of Physical } \\
\text { Education,, Faculty of Physiotherapy, Laboratory of Molecular Biology; } \\
\text { Medical University of Silesia, School of Medicine in Katowice, Department } \\
\text { for Experimental Medicine } \\
\text { Wiaderkiewicz, Jan; Medical University of Silesia, School of Medicine in } \\
\text { Katowice, Department for Experimental Medicine; Rosalind Franklin } \\
\text { University of Medicine and Science, Department of Physiology \& Biophysics } \\
\text { Jędrzejowska-Szypułka, Halina; Medical University of Silesia, School of } \\
\text { Medicine in Katowice, Department of Physiology } \\
\text { Barski, Jarosław; Medical University of Silesia, School of Medicine in } \\
\text { Katowice, Department for Experimental Medicine; Medical University of } \\
\text { Silesia, School of Medicine in Katowice, Department of Physiology } \\
\text { Lewin-Kowalik, Joanna; Medical University of Silesia, School of Medicine in } \\
\text { Katowice, Department of Physiology }\end{array}$ \\
\hline \multicolumn{2}{|l|}{$\begin{array}{r}\text { Is the invited manuscript for } \\
\text { consideration in a Special } \\
\text { Issue? : }\end{array}$} \\
\hline Keyword: & $\begin{array}{l}\text { ketogenic diet, dietary intake < energy regulation, growth, wheat bran, } \\
\text { Insulin-like growth factor } 1\end{array}$ \\
\hline
\end{tabular}

\section{SCHOLARONE ${ }^{m}$




\section{The Modification of the Ketogenic Diet Mitigates Its Stunting Effects in Rodents}

Liśkiewicz, Arkadiusz Damian $^{1,2 *}$; Kasprowska-Liśkiewicz, Daniela ${ }^{2,3}$; Sługocka, Anna ${ }^{1,3}$, Nowacka-Chmielewska, Marta Maria ${ }^{2,3}$; Wiaderkiewicz, Jan ${ }^{3,4}$; Jędrzejowska-Szypułka, Halina ${ }^{1}$; Barski, Jarosław Jerzy ${ }^{1,3}$ and Lewin-Kowalik, Joanna ${ }^{1}$

1 Department of Physiology, School of Medicine in Katowice, Medical University of Silesia,

Katowice, Poland

2 Laboratory of Molecular Biology, Faculty of Physiotherapy, The Jerzy Kukuczka Academy of Physical Education, Katowice, Poland

3 Department for Experimental Medicine, School of Medicine in Katowice, Medical University of Silesia, Katowice, Poland

4 Department of Physiology \& Biophysics, Rosalind Franklin University of Medicine and Science, Chicago, IL, USA

*Corresponding author:

E-mail: adliskiewicz@gmail.com 


\begin{abstract}
The high fat and low carbohydrate ketogenic diet (HFKD) is extensively studied within the fields of numerous diseases, including cancer and neurological disorders. Since most studies incorporate animal models, ensuring the quality of ketogenic rodent diets is important, both in the context of laboratory animal welfare as well as for the accuracy of the obtained results. In this study we implemented a modification to a commonly-used ketogenic rodent chow by replacing non-resorbable cellulose with wheat bran. We assessed the effects of month-long treatment with either the unmodified or the modified HFKD on the growth and development of young male rats. Daily body weight, functional performance, and brain morphometric parameters were assessed to evaluate the influence of both applied diets on rodent development. Our results revealed that the unmodified ketogenic chow induced strong side effects that included weakness, emaciation, and brain undergrowth concomitant to growth inhibition. However, application of the ketogenic chow supplemented with wheat bran suppressed these adverse side effects, which was associated with the restoration of insulin-like growth factor 1 and a decrease in corticosterone levels. We have also shown that the advantageous results of the modified HFKD are not species- or sex-specific. Our data indicate that the proposed HFKD modification even allows for its application in young animals, without causing detrimental side effects.
\end{abstract}

Key words: ketogenic diet; dietary intake; growth; development; brain maturation; wheat bran; insulin-like growth factor 1. 


\section{Introduction}

High-fat, low-carbohydrate HFKDs are widely used in the therapy of epilepsy and are expected to be valuable in the treatment of various disorders (for review see Politi et al. 2011, Baranano and Hartman 2008, Stafstrom and Roh 2012). However concomitant side effects can occur during the HFKD, e.g. kidney stones (Sampath et al. 2007) and delayed somatic growth (Vining et al. 2002, Peterson et al. 2005, Groleau et al. 2014). The last is most evident in children with prolonged clinical use of HFKDs - very young individuals grow especially poorly on the diet (Vining et al. 2002). Recently, various type of dietary interventions such as the modified Atkins diet and the low glycemic index treatment have been performed and different protocols have been proposed to alleviate dietary impoverishment and the discomfort of pediatric patients (Lee et al. 2016). More is now known, but a lot still must be done to reveal the wide therapeutic spectrum of the HFKD and to understand its complex mechanism of action. Greater research is needed and the role of animal studies cannot be underestimated in this matter.

There are numerous studies in this field that are conducted on animal models where laboratory rodents are fed with special ketogenic chows of various compositions. One very popular ketogenic rodent chow used in research is Bio-Serv F3666, which is mainly composed of animal fat. Although this chow effectively induces ketosis and has been used to demonstrate the beneficial health effects of the HFKD (e.g. Nylen et al. 2005, Badman et al. 2009, Van der Auvera et al. 2005), its application is associated with certain side effects. Strong growth inhibition is often described, especially in young animals where this effect can be easily observed (Kennedy et al. 2007, Pissios et al. 2013).

In our previous work, where rats were fed with the HFKD for up to 8 months, we have introduced a modification of a ketogenic chow, prepared according to the Bio-Serv F3666 
recipe, by replacing non-resorbable cellulose with wheat bran (Liskiewicz et al. 2016). Our modification abolished the adverse effects (growth inhibition, general weakness, hair loss) observed after the long-term application of the regular rodent HFKD. The aim of this study was to provide a broader description and evaluation of this observation.

\section{Materials and methods}

All animals were provided by the Animal House of the Center of Experimental Medicine, Medical University of Silesia, Katowice, Poland and were treated in accordance with the 2010/63/EU Directive for animal experiments. This study was approved and monitored by the Local Ethics Committee in Katowice, resolution number 106/2014 of November 19, 2014.

The experiment was performed on male Long Evans rats fed with an unmodified HFKD (KD group, $\mathrm{n}=10)$ or a modified HFKD supplemented with wheat bran $(\mathrm{mKD}, \mathrm{n}=10)$ from postnatal day (PND) 21 for 30 days. Additional males $(n=11)$ were maintained on a standard diet (ST group) as controls. All animals were daily weighed until 50 days of age. BMI was calculated by dividing body weight (grams) by body length squared (square centimeters). Functional analyses were performed on PND43 (locomotor activity by open field; cage-arena $25 \times 25 \mathrm{~cm}$ for mice or $40 \times 40 \mathrm{~cm}$ for rats, 15 min exploration time, TruScan, Coulbourn Instruments, Allentown, PA) and PND44 (skeletal muscular strength measured by grip test; Ugo Basile, Comerio, Italy). At the end of the experiment (PND51) rats were anesthetized (i.p. injection of $100 \mathrm{mg} / \mathrm{kg}$ ketamine plus $10 \mathrm{mg} / \mathrm{kg}$ xylazine), orbital sinus blood samples were collected and animals were euthanized by a transcardiac perfusion with $10 \%$ formalin in PBS. The brains were used for morphometric analysis. Serum samples were used to measure the $\beta$-hydroxybutyrate ( $\beta \mathrm{HB}$; BioVision, Milpitas, CA, USA), IGF-1 (R\&D System, Minneapolis, MN, USA), and corticosterone (CORT, Demeditec diagnostics GmbH, Germany) levels. Additionally three individuals for each group were housed in separate cages 
to measure food and water intake; measurements were performed three times a week. Nonfasting and fasting glucose levels were assessed in these animals (in whole blood collected from the tip of the tail by a glucometer CardioCheck Professional, IN, USA) at PND33 and PND34 respectively.

At the second stage of the experiment, we aimed to evaluate if observed differences in animal growth are sex- or species-specific. For this purpose we involved female Long Evans rats fed with an unmodified (KDf) or modified HFKD (mKDf) or standard rodent chow (STf) starting from PND21 for the next 30 days, $n=6$ in each group. To reveal if the findings can be translated to other species, the males of C57BL/6J mice were subjected to an unmodified (KDM, n=9) or modified HFKD (mKDM, n=6) and standard food (STM, n=6), starting from PND21 for the next 30 days. All animals in the STf, KDf, mKDf, STM, KDM, and mKDM groups were daily weighed until PND50, the open field test was performed on PND43, and the grip test was performed on PND44.

\section{Rodent diets}

The unmodified ketogenic chow was prepared according to the Bio-Serv F3666 HFKD (Frenchtown, NJ, USA) recipe. The modified HFKD contained the same ingredients as the unmodified ketogenic chow except for cellulose, which was replaced with wheat bran. Both HFKDs, as well as the standard rodent chow, were prepared by the local animal food manufacturer Wytwórnia Pasz "Morawski” (Kcynia, Poland). The detailed composition and nutritional profile of all diets used are shown in Tables 1 and 2 respectively.

\section{Morphometry of gross brain parameters}

The dissected rat brains were subjected to morphometric measurements. A digital caliper was used by a blinded observer to obtain the following brain dimensions: (i) length, (ii) width, (iii) 
height of the cerebrum and (iv) cerebellar length, defined as the distance between the apices of cerebellar lobules III and IX at the midline, (v) width qualified as the distance between the left and right paraflocculus, and (vi) height, i.e. the extent between lobules I and IV at the midline. Three measurements of each parameter of every brain were taken and the average value was calculated. The whole brain (cerebrum + cerebellum) and cerebellar mass were obtained. From this data, the Organosomatic Index (organ mass / the whole animal mass * 100) was calculated for the brain and cerebellum by applying the method described by Shoyinka et al. 2008.

\section{Statistical analysis}

Statistica v. 13.1 (Dell Inc., USA) was used for the statistical analysis and GraphPad Prism 5.01 software (GraphPad Software Inc., USA) was used for the preparation of the graphs. The Shaphiro-Wilk test was used to define the Gaussian distribution. In the case of normally distributed data, the parametric one-way ANOVA was followed by the Fisher post hoc test (serum measurements) or the Tukey post hoc test (morphometry of gross brain parameters, open field test, blood glucose levels). Grip test data was compared using the non-parametric Kruskal-Wallis with Dunn's post hoc test. Body mass and food, calorie, and water intake were analyzed by means of two-way ANOVA with the Bonferroni post hoc test. The statistically significant threshold was fixed at $\mathrm{p}>0.05$. The results are presented as mean $\pm \mathrm{SEM}$, except for grip strength data, which are shown as median, 5-95 interquantile range, and min and max.

\section{Results}

The modification of the HFKD improves the growth and functional strength of young male rats 
The animals in the ST group gained weight in a regular manner, while the animals fed with the HFKDs gained significantly less (Fig. 1 A, B). The weight of the animals in the ST group increased about five times (from $38 \mathrm{~g}$ to $210 \mathrm{~g}$ ). The growth of rats in the KD group was strongly inhibited; during 30 days of feeding with the unmodified HFKD, the body weight of the rats decreased from $56 \mathrm{~g}$ on PND21 to $43 \mathrm{~g}$ at the end of the experiment. On the contrary, rats fed with the modified HFKD showed an increase of body mass, starting from $51 \mathrm{~g}$ on PND21 and reaching a final body mass equal to $86 \mathrm{~g}$ on PND50. The weight of the animals in the ST group was higher than the weight of rats in the $\mathrm{KD}$ and $\mathrm{mKD}$ groups, starting from PND27 to the end of the experiment. The animals in the mKD group were significantly heavier than rats in the KD group starting from PND32 $(\mathrm{p}<0.01)$ through the end of the experiment (PND33-PND50 p<0.001). The modification of the HFKD also improved the animals’ BMI (Fig 1 C).

The grip test was performed to assess skeletal muscular strength. Rats fed with an unmodified HFKD were significantly weaker when compared to the $\mathrm{mKD}$ and ST groups (Fig. 1 D). The results did not show a significant difference in the mobility between the KD, mKD, and SD groups, measured as total distance moved or velocity (Fig $1 \mathrm{E}, \mathrm{F}$ ).

\section{The modification of the HFKD improves brain development in young male rats}

Mass measurements revealed that the mean brain and cerebellum mass in the KD group was significantly lower than in the mKD group (Table 3). These organs, in both KD and $\mathrm{mKD}$ groups, were lighter in comparison to the control.

Morphometric studies revealed that all of the measured dimensions of the cerebrum and cerebellum were smaller in the KD group as compared to the ST and $\mathrm{mKD}$ groups (Table 3). A comparison of the measured dimensions between $\mathrm{mKD}$ and ST groups showed 
differences only in cerebral length and width, but not in cerebral height or cerebellar parameters.

\section{Food and water intake, blood $\beta \mathrm{HB}$, and glucose levels in young male rats}

The average consumption of ketogenic chow in mKD group increased about $5.7 \mathrm{~g} /$ day per individual in comparison to the KD group (Fig 2 A). Because of the higher caloric density of the HFKDs in comparison to the standard rodent chow (Table 2), the animals in the mKD group consumed a similar amount of calories as control rats (Fig 2 B). There were significant differences in caloric intake for ST vs. KD groups and among ketogenic groups, where animals from the mKD group consumed significantly more calories (about $42 \mathrm{kcal}$ per day).

Average daily water intake per animal in the ST group was significantly higher than in the $\mathrm{KD}$ and $\mathrm{mKD}$ groups (Fig $2 \mathrm{C}$ ). Daily water consumption was on average $\sim 71 \%$ less in the $\mathrm{KD}$ and $\sim 65 \%$ in the $\mathrm{mKD}$ groups than the ST group. Decreased water intake in animals fed with the HFKD is in line with our earlier observations and is likely a result of (1) reduced food consumption, because rats mostly drink during eating and (2) increased endogenous (mitochondrial) water production due to the intensive metabolism of ketones.

Rats treated with an unmodified and with a modified HFKD respectively had about a 12- and 8-times higher concentration of serum $\beta \mathrm{HB}$ as compared to the control group (Fig $2 \mathrm{D}$ ). In the $\mathrm{KD}$ group, the level of $\beta \mathrm{HB}$ was about 1.5 times higher than in the mKD group. When compared to the control group, the blood levels of non-fasting glucose were significantly decreased in animals fed with the HFKDs and equal in the KD and mKD groups (Fig 2 E). However, fasting glucose did not differ among all groups. We have only observed a statistical decrease in fasting vs. non-fasting glucose within the ST group.

The modification of the HFKD restores serum IGF-1 and CORT levels in young male rats 
The concentration of IGF-1 was significantly decreased in rats from the KD group as compared to the control (Fig 2 F). In contrast, the IGF-1 level in the group with wheat bran supplementation did not significantly differ from the control, but was still higher than in the KD group.

In the KD groups, the serum CORT levels were elevated as compared to the ST group (Fig 2 G). There were no significant differences between the $\mathrm{mKD}$ and the KD or the ST groups.

\section{The beneficial effects of the modified HFKD are not sex- or species- dependent}

The weight of the animals fed with the unmodified formula decreased during the 30 days of treatment; to $76 \%$ of the initial body mass in the case of male rats, to $80 \%$ in the case of female rats (Fig $3 \mathrm{~A}$ ), and to $82 \%$ in the case of mice (Fig 4 A). On the contrary, animals fed with the modified HFKD gained up to $169 \%$ of their initial body mass in the case of male rats, up to $152 \%$ in the case of female rats, and up to $164 \%$ in the case of mice. When expressed as a percent of the initial body mass, the weight gain was similar in the male and female rats independently of the HFKD applied (Fig 3 B). The dimorphic differences in weight gain were observed only between ST and STf groups - from the 19th to the 30th day of the experiment (PND39-50) (Fig 3 B) - where males fed with regular rodent chow grew about $39 \%$ more than females. The weight gain pattern was also similar in the rats and mice (Fig 4 B). It is worth noticing that the rats fed with a modified HFKD weighed $41 \%$ of the body mass of age-matched rats fed with the standard diet, while mice reached $82 \%$ of the body mass of age-matched controls. This difference is associated with heterogenous patterns of body weight gain in mice and rats; namely, between PND21 and PND50 the weight of rats fed with standard rodent chow increased up to $500 \%$ of their initial body mass, while in case of mice it is only around $200 \%$. 
As revealed by the grip test, the changes in the functional strength of female rats (Fig. 3 C) and mice (Fig. 4 C) showed similar patterns as those observed in the case of male rats. In the open field test, the results did not show a significant difference in mobility between the KDf, mKDf, and STf groups, measured as the total distance moved or the velocity (Fig $3 \mathrm{D}$, E); similar results were observed for the male rats. However, an analysis of locomotor functions revealed the impaired movement abilities and speed of mice treated with an unmodified HFKD. The total distance traveled by mice from the KDM group was shorter compared to animals fed with mKDM or ST chow (Fig. 4 D). The mean velocity of the animals in the KDM group was lower than in the mKDM and STM groups (Fig. 4 E).

\section{Discussion}

In the present study it was shown that the addition of wheat bran into an almost fully animal fat-based ketogenic chow has significantly mitigated its negative effects on the growth and development of rats. The observed negative effects of the unmodified ketogenic chow were confirmed in consultations with other researchers in the field and in the scientific literature. For instance, it was shown that the F3666 diet inhibited the growth of 21-day-old rats (Nylen et al. 2005) and mice (Badman et al. 2009) when administered for 10 and 49 days, respectively. In other studies performed on 7-8-week-old mice, the F3666 diet drastically reduced their body weight (Kennedy et al. 2007) or prevented weight gain (Jornayvaz et al. 2010), which was associated with decreased lean body mass in both cases. Some researchers also emphasized the detrimental impact of the F3666 diet: "Due to some problems with weight loss in animals in the KD group, these animals were fed a mixed chow (...). However, one mouse in the KD group refused food intake and died despite attempts of feeding via gavage" (Van der Auwera et al. 2005). 
The animals fed with the modified HFKD grew and gained weight in contrast to the animals fed with an unmodified formula, whose growth was inhibited, although much slower than the control group. This effect was not sex-dependent. The magnitude of the beneficial effects of the applied modification was similar in male and female rats, but the dimorphic difference in weight gain were negated during HFKD and the administration of wheat bran couldn't overcome this issue. Moreover, some authors emphasize the distinct growth pattern among adult males and females, which probably results from gender differences in hormonal status (Bielohuby et al. 2011, Zengin et al. 2016). In our study, very young animals were examined and the lack of differences in weight gain may result from similar hormonal conditions in pre-pubertal individuals, which could confirm the distinct role of sex hormones in growth during ketogenic treatment as proposed earlier (Bielohuby et al. 2011). The growth pattern is also not species-dependent, but if the weight gain of animals is considered as a percent of the body mass of age-matched controls, the effect of the modified diet can be considered as larger in mice when compared to rats. However, this conclusion must be considered carefully because rats grow more intensively than do mice, irrespective of the applied diet. The modification of the ketogenic chow also improved the functional strength parameters. All animals fed with the unmodified HFKD had significantly lower grip strength of the forepaws, while the modified formula restored this weakness. Further, mice fed with the unmodified HFKD had decreased locomotor activity, but the locomotor activity parameters did not differ between rat groups. It is hard to explain the origin of this interspecies difference, but it can be attributed to differences in the developmental pattern of the brain in rats and mice (Pressler and Auvin 2013) and the interspecies differences in the susceptibility to malnutrition (Jousse et al. 2014). The nutrient deficiency related to the unmodified HFKD may have a more severe impact on the motor abilities of mice than of rats. The observed difference in locomotor activity reflects previous studies: it was shown that the 
HFKD (Bio-Serv F3666) decreases physical efficiency in mice (Asrih et al. 2015), but not in rats (Ruskin et al. 2013, Thio et al. 2010). Importantly, we have shown that the modification of the ketogenic chow restores the locomotor activity of mice to control levels.

Both HFKDs affected brain development, but the unmodified diet had a more deleterious effect than the formula that contained wheat bran. These positive properties of the modified HFKD were especially demonstrated by the relatively normal growth of the cerebellum rather than the more nutrient-demanding cerebrum. This idea might be supported by the fact that the cerebellum of underfed rats manifests the strongest growth power, which is higher than in other parts of the brain (Stewart 1918). Intriguing data was also noted by assessing brain mass. The weight of the brain as well as the cerebellum was negatively affected by both HFKDs, but the calculated brain/body mass index was elevated with respect to the body. These results are in line with some earlier studies and support the conclusion that the brain grows nonlinearly with respect to the body (Martins et al. 2011), and that an elevated brain/body mass index is an indicator of malnutrition.

Furthermore, the addition of wheat bran to the HFKD normalized IGF-1 and decreased CORT levels in the sera of fed rats. IGF-1 is crucial for normal somatic development and its depletion is associated with the delayed growth of children treated with the HFKD (Vining et al. 2002, Peterson et al. 2005, Groleau et al. 2014), thus it is important to maintain its level during HFKD. On the contrary, oversecreted CORT affects nearly every organ and tissue in the body and its elevated level may disturb normal development in young individuals (Sapolsky et al. 2000). Thus, it seems to be important to reduce CORT levels. This study showed that in animal models, this can be achieved by supplementing the ketogenic chow with wheat bran. However, the CORT levels in rats fed with the modified HFKD were still higher than in control animals, probably because of the permanent overproduction of glucocorticoids typical of fasting-mimicking diets (Choi et al. 2016). The lowered level in the 
blood $\beta \mathrm{HB}$ in rats fed with the modified HFKD as compared to the unmodified formula may be related to the reduction of CORT levels, because this hormone stimulates ketogenesis (Mittendorfer and Klein 2014).

Importantly, elevated $\beta \mathrm{HB}$ concentration, low glucose level, and preserved gluconeogenic mechanisms confirm that the addition of wheat bran does not affect the metabolic status typical of the application of the HFKD. In summary, we postulate that increased IGF-1 levels and decreased CORT production can be responsible for the beneficial effects of the applied modification of the ketogenic chow. The changes in IGF-1 and CORT levels could simply result from enhanced food consumption or the action of different active compounds occurring in wheat bran. We observed that animals fed with the modified formula consumed more chow than did individuals fed with the unmodified HFKD, ensuring the appropriate caloric intake. Moreover, considering that rodents naturally consume high amounts of dietary fibers, it is probable that the addition of grains (wheat bran) into a lardbased mixture improved the gastrointestinal tolerability of the HFKD. Additionally, because of the enhanced food consumption the animals also received more vitamins and microelements, which seemingly contributed to the beneficial effects of the modified HFKD. Thus the positive effects of our modified diet may also be related to the supply of some naturally-occurring compounds of wheat bran (e.g. antioxidants and vitamins), which are scarce in unmodified ketogenic rodent chow (Stevenson et al. 2012). For example, an important role may be assigned to betaine, in which wheat bran is particularly abundant as it contains $1.34 \mathrm{~g}$ of betaine in every $100 \mathrm{~g}$ (Zeisel et al. 2003). Human data showed that the daily supplementation of $1.25 \mathrm{~g}$ of betaine (about $0.015 \mathrm{~g} / \mathrm{kg}$ ) enhanced the anabolic endocrine profile by increasing blood IGF-1 levels along with a concomitant decrease in cortisol (Ganesan et al. 2011, Apicella et al. 2013). Here we have observed the similar endocrine status of animals when wheat bran was supplemented, which suggest a betaine- 
dependent mechanism. Although further research is needed to support this finding, it seems to be reasonable, especially considering the fact that choline acts as a betaine precursor. It has been shown previously that supplementation with choline mitigates the negative side effects of the HFKD prepared according to the Bio-Serv F3666 recipe (Pissois et al. 2013, Schugar et al. 2013). Pissios et al. showed that the supplementation of the HFKD with choline or methionine improved the weight gain of mice fed with the HFKD (Pissios et al. 2013). Schugar et al. 2013 performed a modification of the Bio-Serv F3666-like chow by adding an extra $5 \mathrm{~g} / \mathrm{kg}$ of choline and/or delivering an extra 5 percent of calories from proteins. Both modifications resulted in the improved growth of the animals. In the present study, the supplementation of the HFKD with wheat bran alters the calorie income from proteins by only $0.14 \%$ (Balandrán-Quintana et al. 2015). Thus the positive effects of our modification likely cannot be explained by a greater amount of protein in the diet. However, choline and betaine occurring in wheat bran can significantly contribute to the beneficial effect of the modified HFKD. Wheat bran contains about $0.03 \mathrm{~g}$ of B vitamins per $100 \mathrm{~g}$ (Stevenson et al. 2012); thus individual rats fed with a modified HFKD consumed these elements about $0.2 \mathrm{mg}$ more per day than did the other rats maintained on classical ketogenic chow. For example, the positive effect of wheat bran due to the administration of B vitamins may be justified by the concomitant increased supply of thiamine, because its administration in modified ketogenic groups might support an increase in IGF-1 with a simultaneous decrease in CORT (Molina et al. 1996). Further, the cerebellar undergrowth observed only in rats fed with the more scarce HFKD not supplemented with wheat bran may underlie the mentioned role of thiamine as the lack of this vitamin particularly results in cerebellar malformation (Mulholland 2006).

The composition of the ketogenic rodent chow used in the present study is relatively impoverished and may not reflect the present clinical approach well. The use of research diets containing mostly animal fat (similar to Bio-Serv F3666) may be justified historically. Such 
formulas may have better reflected clinical practice in the past, when patients were often sustained on the classic long-chain triglyceride HFKD based on products of an animal origin. Currently, the modified Atkins diet is more widely recommended by clinicians due to its less restrictive manner and greater heterogeneity (Kossoff et al. 2013), thus more complex animal chows may reflect this nutritional approach in a better way. Nevertheless, it should be noted that the classical HFKD is still recommended for infants under the age of 2 and for those receiving formula-only nutrition (e.g., gastrostomy tube-fed) due to its better efficiency in these cases (Kossoff et al. 2013).

In summary, the present study shows that the supplementation of the HFKD with wheat bran alleviates its negative effects on young animals in terms of growth inhibition, functional strength parameters, and cerebral and cerebellar development. The modification also affects the serum levels of IGF-1 and CORT. The beneficial effects are likely associated with increased food consumption, which results in the higher intake of calories, vitamins, and minerals. The proposed modification is easy and relatively cheap, thus researchers should be able to freely use it in future HFKD studies. The study sheds light on the importance of the dietary diversity of HFKDs used in young patients, which may alleviate some unintended side effects typical of this therapeutic approach.

\section{Acknowledgment}

This work was supported by Medical University of Silesia, grant no. KNW-1-051/K/4/0. The authors declare that there is no conflict of interest associated with this manuscript. 


\section{References}

Apicella, J.M., Lee, E.C., Bailey, B.L., Saenz, C., Anderson, J.M., Craig, S.A.S., Kraemer, W.J., Volek, J.S., and Maresh, C.M. 2013. Betaine supplementation enhances anabolic endocrine and Akt signaling in response to acute bouts of exercise. Eur. J. Appl. Physiol. 113(3): 793-802. doi:10.1007/s00421-012-2492-8.

Asrih, M., Altirriba, J., Rohner-Jeanrenaud, F., and Jornayvaz, F.R. 2015. Ketogenic diet impairs FGF21 signaling and promotes differential inflammatory responses in the liver and white adipose tissue. PLoS One, 10(5): e0126364.

doi:10.1371/journal.pone.0126364.

Badman, M.K., Kennedy, A.R., Adams, A.C., Pissios, P., and Maratos-Flier, E. 2009. A very low carbohydrate ketogenic diet improves glucose tolerance in ob/ob mice independently of weight loss. Am. J. Physiol. Endocrinol. Metab. 297(5): E1197-204. doi:10.1152/ajpendo.00357.2009.

Balandrán-Quintana, R.R., Mercado-Ruiz, J.N., and Mendoza-Wilson, A.M. 2015. Wheat Bran Proteins: A Review of Their Uses and Potential. Food Rev. Int. 31(3): 279-293. doi:10.1080/87559129.2015.1015137.

Baranano, K.W., and Hartman, A.L. 2008. The ketogenic diet: uses in epilepsy and other neurologic illnesses. Curr. Treat. Options Neurol. 10(6): 410-419.

Bielohuby, M., Sawitzky, M., Stoehr, B.J.M., Stock, P., Menhofer, D., Ebensing, S., Bjerre, M., Frystyk, J., Binder, G., Strasburger, C., Wu, Z., Christ, B., Hoeflich, A., and Bidlingmaier, M. 2011. Lack of dietary carbohydrates induces hepatic growth hormone (GH) resistance in rats. Endocrinology, 152(5): 1948-1960. doi:10.1210/en.2010-1423.

Choi, I.Y., Piccio, L., Childress, P., Bollman, B., Ghosh, A., Brandhorst, S., Suarez, J., Michalsen, A., Cross, A.H., Morgan, T.E., Wei, M., Paul, F., Bock, M., and Longo, V.D. 2016. A Diet Mimicking Fasting Promotes Regeneration and Reduces Autoimmunity and Multiple Sclerosis Symptoms. Cell Rep. 15(10): 2136-2146. doi:10.1016/j.celrep.2016.05.009.

Ganesan, B., Anandan, R., and Lakshmanan, P.T. 2011. Studies on the protective effects of betaine against oxidative damage during experimentally induced restraint stress in Wistar albino rats. Cell Stress Chaperones, 16(6): 641-652. doi:10.1007/s12192-011-0276-4.

Groleau, V., Schall, J.I., Stallings, V.A., and Bergqvist, C.A. 2014. Long-term impact of the ketogenic diet on growth and resting energy expenditure in children with intractable epilepsy. Dev. Med. Child Neurol. 56(9): 898-904. doi:10.1111/dmcn.12462.

Jornayvaz, F.R., Jurczak, M.J., Lee, H.-Y., Birkenfeld, A.L., Frederick, D.W., Zhang, D., Zhang, X.-M., Samuel, V.T., and Shulman, G.I. 2010. A high-fat, ketogenic diet causes hepatic insulin resistance in mice, despite increasing energy expenditure and preventing 
weight gain. Am. J. Physiol. Endocrinol. Metab. 299(5): E808-815. doi:10.1152/ajpendo.00361.2010.

Jousse, C., Muranishi, Y., Parry, L., Montaurier, C., Even, P., Launay, J.-M., Carraro, V., Maurin, A.-C., Averous, J., Chaveroux, C., Bruhat, A., Mallet, J., Morio, B., and Fafournoux, P. 2014. Perinatal protein malnutrition affects mitochondrial function in adult and results in a resistance to high fat diet-induced obesity. PLoS One, 9(8): e104896. doi:10.1371/journal.pone.0104896.

Kennedy, A.R., Pissios, P., Otu, H., Roberson, R., Xue, B., Asakura, K., Furukawa, N., Marino, F.E., Liu, F.-F., Kahn, B.B., Libermann, T.A., and Maratos-Flier, E. 2007. A high-fat, ketogenic diet induces a unique metabolic state in mice. Am. J. Physiol. Endocrinol. Metab. 292(6): E1724--39. doi:10.1152/ajpendo.00717.2006.

Kossoff, E.H., Cervenka, M.C., Henry, B.J., Haney, C.A., and Turner, Z. 2013. A decade of the modified Atkins diet (2003-2013): Results, insights, and future directions. Epilepsy Behav. 29(3): 437-442. doi:10.1016/j.yebeh.2013.09.032.

Lee, E., Kang, H.-C., and Kim, H.D. 2016. Ketogenic Diet for Children with Epilepsy: A Practical Meal Plan in a Hospital. Clin. Nutr. Res. 5(1): 60-63. doi:10.7762/cnr.2016.5.1.60.

Liskiewicz, A.D., Kasprowska, D., Wojakowska, A., Polanski, K., Lewin-Kowalik, J., Kotulska, K., and Jedrzejowska-Szypulka, H. 2016. Long-term High Fat Ketogenic Diet Promotes Renal Tumor Growth in a Rat Model of Tuberous Sclerosis. Sci. Rep. 6: 21807. doi:10.1038/srep21807.

Martins, V.J.B., Toledo Florêncio, T.M.M., Grillo, L.P., Franco, M. do C.P., Martins, P.A., Clemente, A.P.G., Santos, C.D.L., Vieria, M. de F.A., and Sawaya, A.L. 2011. Longlasting effects of undernutrition. Int. J. Environ. Res. Public Health 8(6): 1817-1846. doi:10.3390/ijerph8061817.

Mittendorfer, B., and Klein, S. 2014. Absence of leptin triggers type 1 diabetes. Nat. Med. 20(7): 705-706. doi:10.1038/nm.3629.

Molina, P.E., Fan, J., Boxer, R., Gelato, M.C., Lang, C.H., and Abumrad, N.N. 1996 Modulation of insulin-like growth factor-I: A specific role for vitamin B 1 (thiamine). $\mathrm{J}$ Nutr. Biochem. 7(4): 217-213. doi:10.1016/0955-2863(96)00011-3.

Mulholland, P.J. 2006. Susceptibility of the cerebellum to thiamine deficiency. Cerebellum, 5(1): 55-63. doi:10.1080/14734220600551707.

Nylen, K., Likhodii, S., Abdelmalik, P.A., Clarke, J., and Burnham, W.M. 2005. A Comparison of the Ability of a 4:1 Ketogenic Diet and a 6.3:1 Ketogenic Diet to Elevate Seizure Thresholds in Adult and Young Rats. Epilepsia, 46(8): 1198-1204. 10.1111/j.1528-1167.2005.71204.x

Peterson, S.J., Tangney, C.C., Pimentel-Zablah, E.M., Hjelmgren, B., Booth, G., and BerryKravis, E. 2005. Changes in growth and seizure reduction in children on the ketogenic 
diet as a treatment for intractable epilepsy. J. Am. Diet. Assoc. 105(5): 718-725. doi:10.1016/j.jada.2005.02.009.

Pissios, P., Hong, S., Kennedy, A.R., Prasad, D., Liu, F.F., and Maratos-Flier, E. 2013. Methionine and choline regulate the metabolic phenotype of a ketogenic diet. Mol. Metab. 2(3): 306-313. doi:10.1016/j.molmet.2013.07.003.

Politi, K., Shemer-Meiri, L., Shuper, A., and Aharoni, S. 2011. The ketogenic diet 2011: how it works. Epilepsy Res. Treat. 2011: 963637. doi:10.1155/2011/963637.

Pressler, R., and Auvin, S. 2013. Comparison of Brain Maturation among Species: An Example in Translational Research Suggesting the Possible Use of Bumetanide in Newborn. Front. Neurol. 4: 36. doi:10.3389/fneur.2013.00036.

Ruskin, D.N., Suter, T.A., Ross, J.L., and Masino, S.A. 2013. Ketogenic diets and thermal pain: dissociation of hypoalgesia, elevated ketones, and lowered glucose in rats. J. Pain, 14(5): 467-74. doi: 10.1016/j.jpain.2012.12.015.

Sampath, A., Kossoff, E.H., Furth, S.L., Pyzik, P.L., and Vining, E.P.G. 2007. Kidney stones and the ketogenic diet: risk factors and prevention. J. Child Neurol. 22(4): 375-378. doi:10.1177/0883073807301926.

Sapolsky, R.M., Romero, L.M., and Munck, A.U. 2000. How do glucocorticoids influence stress responses? Integrating permissive, suppressive, stimulatory, and preparative actions. Endocr. Rev. 21(1): 55-89. doi:10.1210/edrv.21.1.0389.

Schugar, R.C., Huang, X., Moll, A.R., Brunt, E.M., and Crawford, P.A. 2013. Role of Choline Deficiency in the Fatty Liver Phenotype of Mice Fed a Low Protein, Very Low Carbohydrate Ketogenic Diet. PLoS One, 8(8): e74806. doi:10.1371/journal.pone.0074806.

Shoyinka, S.V.O., Obidike, I.R., and Ndumnego, C.O. 2008. Effect of iodine supplementation on thyroid and testicular morphology and function in euthyroid rats. Vet. Res. Commun. 32(8): 635-645. doi:10.1007/s11259-008-9065-7.

Stafstrom, C.E., and Rho, J.M. 2012. The ketogenic diet as a treatment paradigm for diverse neurological disorders. Front. Pharmacol. 3: 1-8. doi:10.3389/fphar.2012.00059.

Stevenson, L., Phillips, F., O’Sullivan, K., and Walton, J. 2012. Wheat bran: its composition and benefits to health, a European perspective. Int. J. Food Sci. Nutr. 63(8): 1001-1013. doi:10.3109/09637486.2012.687366.

Stewart, C.A. 1918. Weights of various parts of the brain in normal and underfed albino rats at different ages. J. Comp. Neurol. 29(5): 511-528. doi:10.1002/cne.900290504.

Thio, L.L., Rensing, N., Maloney, S., Wozniak, D.F., Xiong, C., and Yamada, K.A. 2010. A ketogenic diet does not impair rat behavior or long-term potentiation. Epilepsia, 51(8): 1619-1623. doi:10.1111/j.1528-1167.2009.02515.x. 
Van der Auwera, I., Wera, S., Van Leuven, F., and Henderson, S.T. 2005. A ketogenic diet reduces amyloid beta 40 and 42 in a mouse model of Alzheimer's disease. Nutr. Metab. 2:28. doi:10.1186/1743-7075-2-28.

Vining, E.P.G., Pyzik, P., McGrogan, J., Hladky, H., Anand, A., Kriegler, S., and Freeman, J.M. 2002. Growth of children on the ketogenic diet. Dev. Med. Child Neurol. 44(12): 796-802.

Zeisel, S.H., Mar, M.-H., Howe, J.C., and Holden, J.M. 2003. Concentrations of cholinecontaining compounds and betaine in common foods. J. Nutr. 133(1): 1302-1307. doi:10.1080/07347330903549820.

Zengin, A., Kropp, B., Chevalier, Y., Junnila, R., Sustarsic, E., Herbach, N., Fanelli, F., Mezzullo, M., Milz, S., Bidlingmaier, M., and Bielohuby, M. 2016. Low-carbohydrate, high-fat diets have sex-specific effects on bone health in rats. Eur. J. Nutr. 55(7): 23072320. doi:10.1007/s00394-015-1040-9. 
Table 1. Comparison of the composition of standard rodent chow, unmodified, and modified HFKDs used in the present study and Bio-Serv F3666 ketogenic rodent chow.

\begin{tabular}{|c|c|c|c|c|}
\hline Ingredients & $\begin{array}{l}\text { Standard chow } \\
(\mathrm{g} / \mathrm{kg})\end{array}$ & $\begin{array}{l}\text { Unmodified } \\
\text { HFKD }(\mathrm{g} / \mathrm{kg})\end{array}$ & $\begin{array}{c}\text { Modified HFKD } \\
(\mathrm{g} / \mathrm{kg})\end{array}$ & $\begin{array}{c}\text { BiosServ F3666 } \\
(\mathrm{g} / \mathrm{kg})\end{array}$ \\
\hline Lard & - & 478.09 & 478.09 & 475 \\
\hline Butter fat & - & 200 & 200 & 199.5 \\
\hline Corn oil & - & 110 & 110 & 114 \\
\hline Casein & - & 95 & 95 & 95 \\
\hline Dextrose & - & 7.5 & 7.5 & 7.6 \\
\hline Cellulose & - & 50 & - & 50 \\
\hline Wheat bran & 200 & - & 50 & - \\
\hline Wheat & 364 & - & - & - \\
\hline Corn & 200 & - & - & - \\
\hline Dried whey & 30 & - & - & - \\
\hline Soybean meal & 170 & - & - & - \\
\hline Mineral and & 36 & 59.41 & 59.41 & 58.9 \\
\hline Vitamin mix & & & & \\
\hline
\end{tabular}


Table 2. Macronutrient composition of rodent diets used in this study. Values represent caloric content per gram of nutrient. The ratio was calculated by dividing the fat mass by the sum of the carbohydrates and proteins.

\begin{tabular}{lcclll}
\hline \multicolumn{1}{c}{ Diet } & Carb & Fat & Protein & $\mathrm{kcal} / \mathrm{g}$ & Ratio \\
& $\mathrm{kcal} / \mathrm{g}$ & $\mathrm{kcal} / \mathrm{g}$ & $\mathrm{kcal} / \mathrm{g}$ & & \\
& & & & & \\
\hline Standard & 2.6 & 0.25 & 0.77 & 3.57 & 0.03 \\
Unmodified HFKD & 0.13 & 6.8 & 0.32 & 7.25 & 6.1 \\
& & & & & \\
Modified HFKD & 0.19 & 6.8 & 0.34 & 7.33 & 5.6 \\
\hline
\end{tabular}


Table 3. Brain-to-body mass ratio and gross brain morphometry data of rats subjected to the unmodified HFKD (KD group), the modified HFKD supplemented with wheat bran (mKD group), or standard rodent chow (ST group) $\left({ }^{*} \mathrm{p}<0.05, * * \mathrm{p}<0.01, * * * \mathrm{p}<0.001\right.$, ns - not significant, when compared among ST and ketogenic groups).

\begin{tabular}{|c|c|c|c|c|}
\hline & ST & $\mathrm{KD}$ & $\mathrm{mKD}$ & KD vs. mKD \\
\hline Brain weight $(g)$ & $1.51 \pm 0.01$ & $1.22 \pm 0.02^{* * *}$ & $1.37 \pm 0.01^{* * *}$ & $\mathrm{p}<0.001$ \\
\hline Cerebellar weight $(g)$ & $0.25 \pm 0.01$ & $0.2 \pm 0.01^{* * *}$ & $0.23 \pm 0.01^{* * *}$ & $\mathrm{p}<0.001$ \\
\hline Brain/body weight index & $0.7 \pm 0.02$ & $2.9 \pm 0.11^{* * *}$ & $1.6 \pm 0.1^{*}$ & $\mathrm{p}<0.001$ \\
\hline Cerebellar/body weight index & $0.12 \pm 0.01$ & $0.47 \pm 0.02^{* * * *}$ & $0.26 \pm 0.01^{* * * *}$ & $\mathrm{p}<0.001$ \\
\hline Cerebral length $(\mathrm{mm})$ & $13.93 \pm 0.1$ & $12.27 \pm 0.2^{* * *}$ & $13.1 \pm 0.08^{* *}$ & $\mathrm{p}<0.01$ \\
\hline Cerebral width (mm) & $11.57 \pm 0.07$ & $10.52 \pm 0.09^{* * *}$ & $11.29 \pm 0.1^{* *}$ & $\mathrm{p}<0.001$ \\
\hline Cerebral height $(\mathrm{mm})$ & $8.9 \pm 0.08$ & $8.1 \pm 0.12^{* * *}$ & $8.8 \pm 0.12$ & $\mathrm{p}<0.001$ \\
\hline Cerebellar length $(\mathrm{mm})$ & $6.13 \pm 0.09$ & $5.3 \pm 0.07^{* * *}$ & $5.87 \pm 0.06^{\mathrm{ns}}$ & $\mathrm{p}<0.001$ \\
\hline Cerebellar width $(\mathrm{mm})$ & $11.57 \pm 0.07$ & $10.52 \pm 0.09^{* * *}$ & $11.59 \pm 0.01^{\mathrm{ns}}$ & $\mathrm{p}<001$ \\
\hline Cerebellar height $(\mathrm{mm})$ & $8.92 \pm 0.08$ & $8.11 \pm 0.12^{* * *}$ & $8.82 \pm 0.12^{\mathrm{ns}}$ & $\mathrm{p}<0.01$ \\
\hline
\end{tabular}


Fig 1. Thirty-day body mass measurement of male rats (A) maintained on the unmodified HFKD (KD group) or the modified HFKD supplemented with wheat bran (mKD) compared to controls fed with standard rodent chow (ST). The growth of animals fed by unmodified HFKD was stopped, whereas animals treated with the modified formula grew, but not as fast as the control animals $(\# \# \mathrm{p}<0.01, \# \# \# \mathrm{p}<0.001$ for $\mathrm{KD}$ vs $\mathrm{mKD}$ groups). (B) Documentation of rat phenotypes treated with particular chow. Body mass index (C) and functional parameters such as muscular strength (D) and locomotor activity (E, F) were measured and compared among groups $(* * \mathrm{p}<0.01, * * * \mathrm{p}<0.001)$.

Fig 2. (A) Food intake, (B) calorie intake, and (C) water intake per day in rats subjected to the unmodified HFKD (KD group), the modified HFKD supplemented with wheat bran (mKD group), or standard rodent chow (ST group) (\#\#p<0.01, \#\#\#p<0.001 for KD vs mKD groups). (D) Concentration of serum $\beta$-hydroxybutyrate, (E) non-fasting and overnight fasting glucose, (F) insulin-like growth factor 1 , and $(\mathrm{G})$ corticosterone $(*<\mathrm{p}<0.05, * * \mathrm{p}<0.01$, $* * * \mathrm{p}<0.001)$

Fig 3. Parameters analyzed in female rats subjected to the unmodified HFKD (KDf group), the modified HFKD supplemented with wheat bran (mKDf group), or standard rodent chow (STf group). (A) The growth of female rats fed with the unmodified HFKD was stopped, whereas animals treated with the modified formula grew, but still not as fast as control animals (\#p<0.05, \#\#p<0.01, \#\#\#p<0.001 for KDf vs mKDf groups). (B) Intersex comparison of weight gain revealed that males fed with standard rodent chow grew more intensively than females (since 39 to 50 PND), but this dimorphic difference was not observed within ketogenic groups. (C) Grip strength decreased in female rats from KD group whereas animals maintained on modified HFKD did not differ as compared to controls. Functional parameters 
such muscular strength (D) and locomotor activity (E, F) were measured and compared among groups $(* \mathrm{p}<0.05, * * * \mathrm{p}<0.001)$.

Fig 4. Parameters analyzed in male mice subjected to the unmodified HFKD (KDM group), the modified HFKD supplemented with wheat bran (mKDM group) or standard rodent chow (STM group). (A) Growth of mice fed by unmodified HFKD was stopped whereas animals treated with modified formula grew, but not as fast as the control animals $(\# \# \mathrm{p}<0.01$, $\# \# \#$ p $<0.001$ for KDM vs mKDM. (B) An interspecies comparison of weight gain among male rats and mice revealed that rats grew more intensively than mice independently of the applied diet. Functional parameters such as (D) muscular strength and (E, F) locomotor activity were measured and compared among groups $(* \mathrm{p}<0.05, * * \mathrm{p}<0.01, * * * \mathrm{p}<0.001)$. 

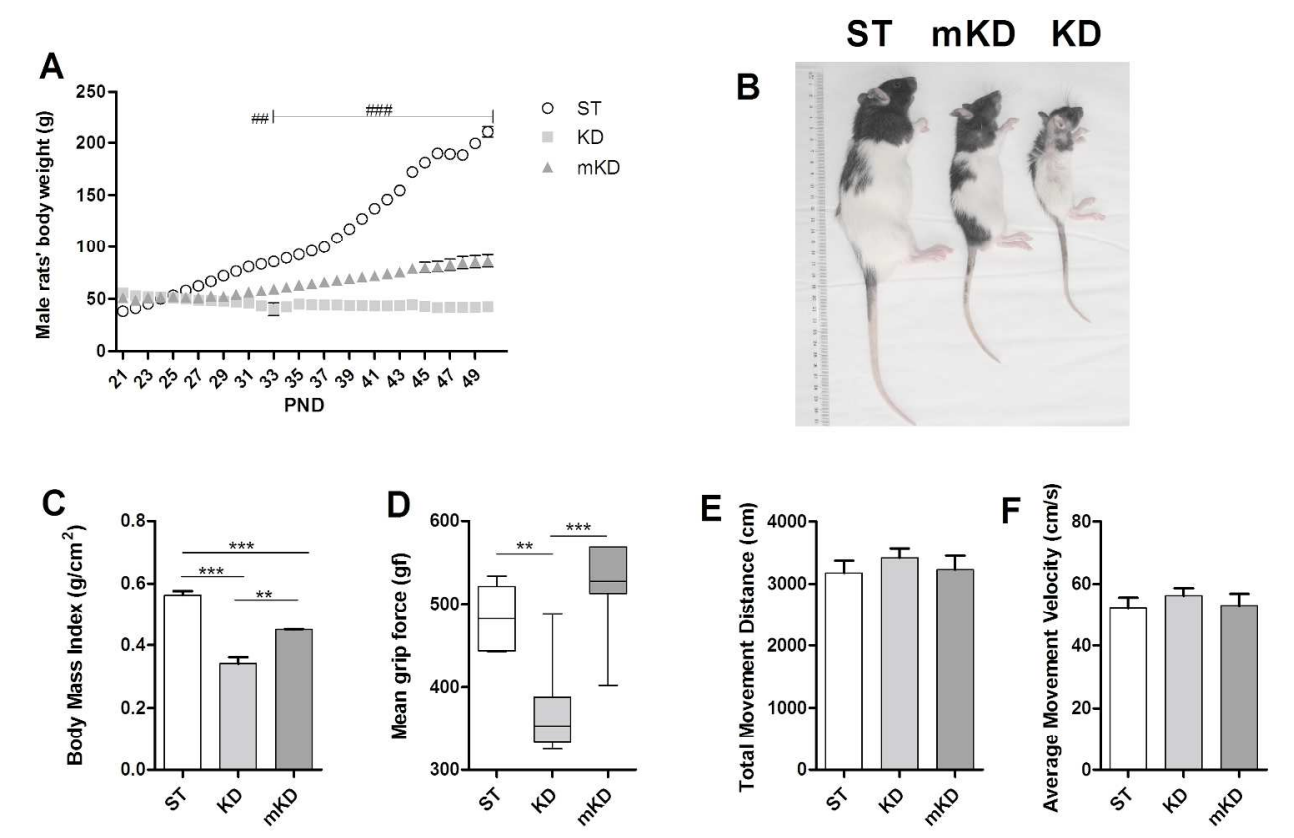

Figure 1

$254 \times 169 \mathrm{~mm}(300 \times 300 \mathrm{DPI})$ 

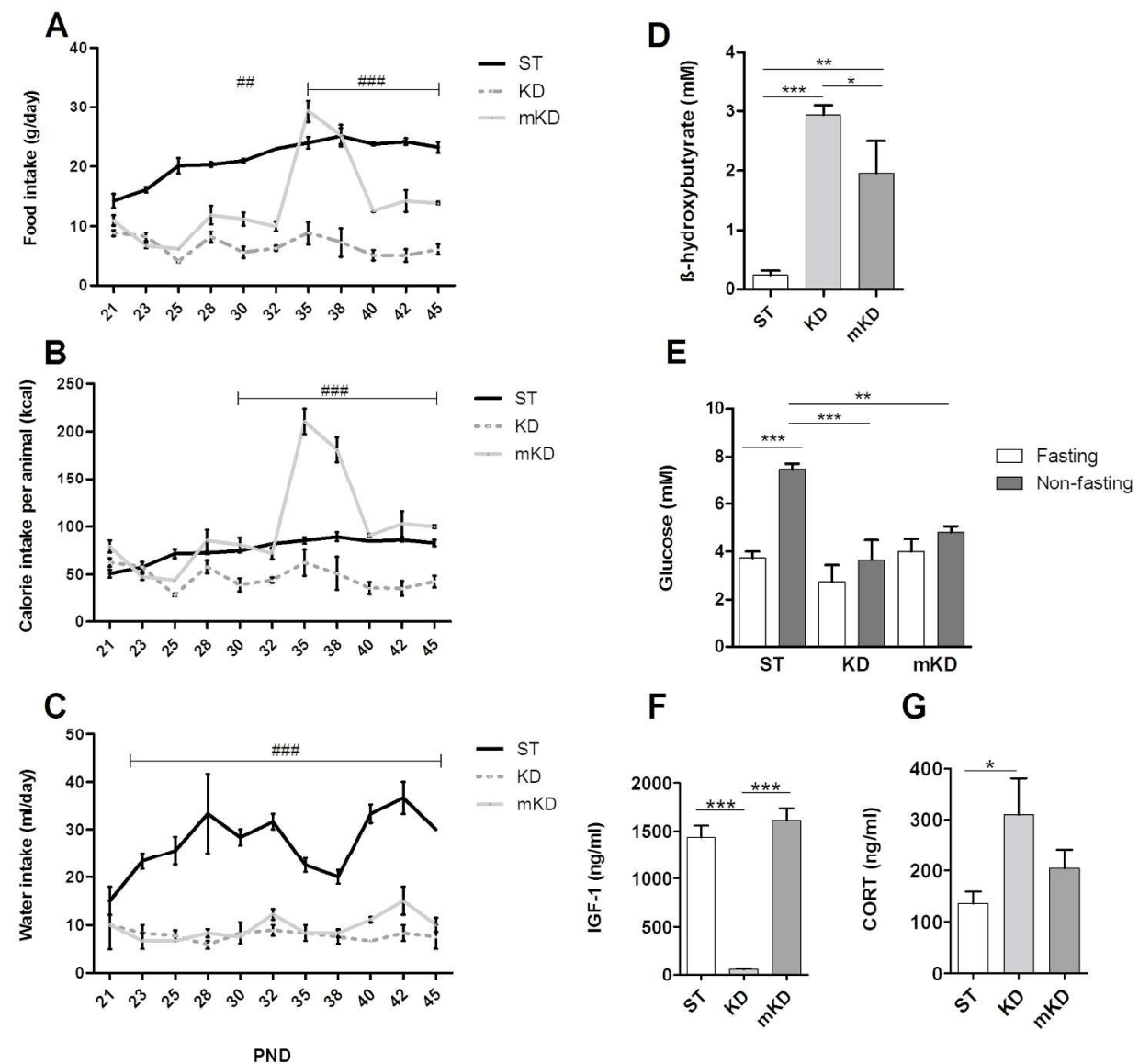

Figure 2

$196 \times 184 \mathrm{~mm}(300 \times 300$ DPI $)$ 

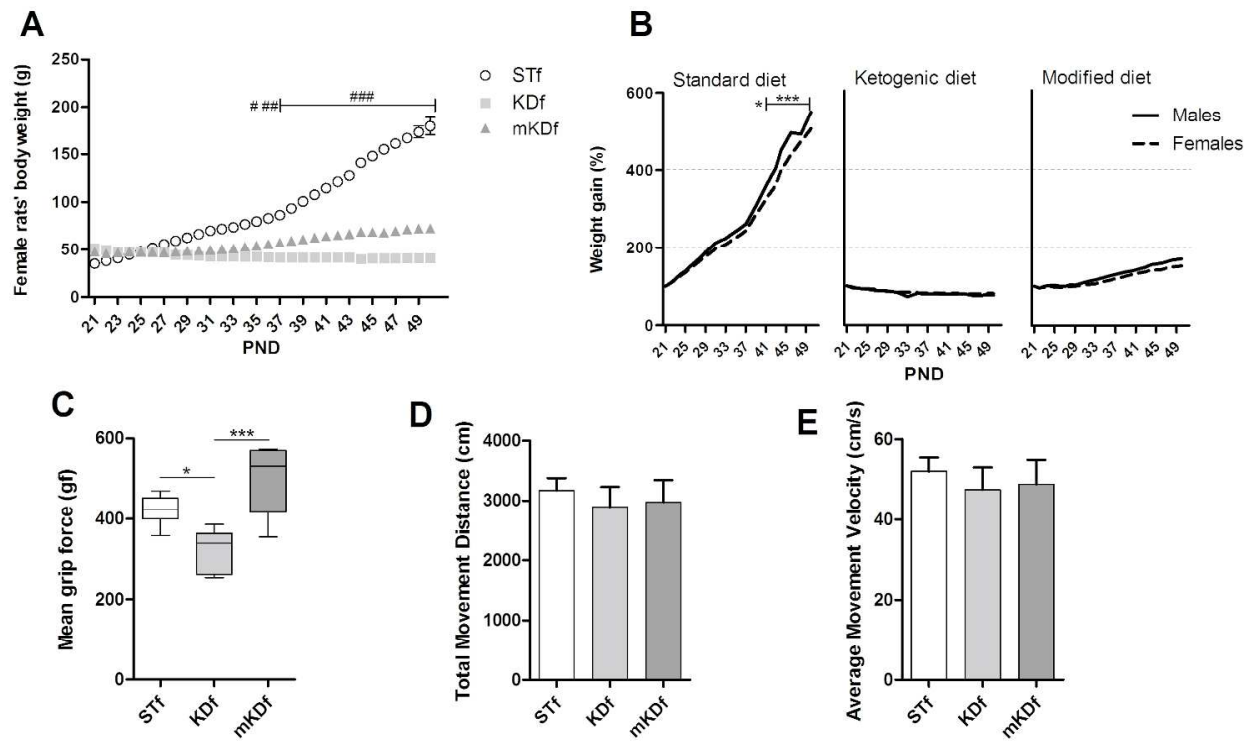

D

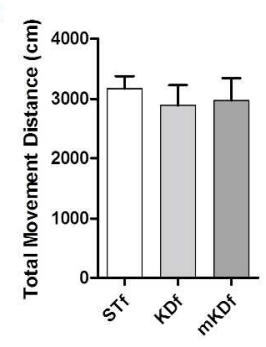

E

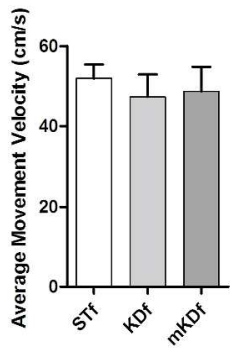

Figure 3

$262 \times 158 \mathrm{~mm}(300 \times 300 \mathrm{DPI})$ 

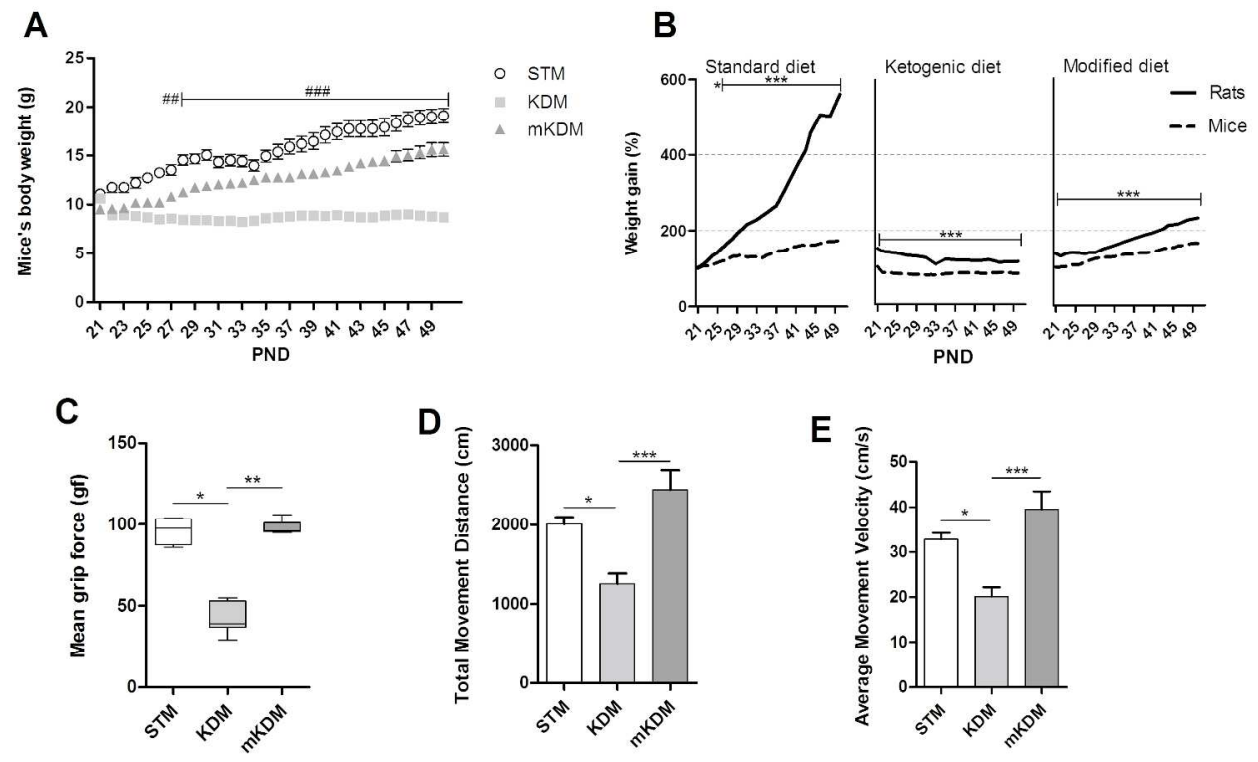

Figure 4

$258 \times 159 \mathrm{~mm}(300 \times 300 \mathrm{DPI})$ 